\title{
Power Control with Nearest Neighbor Nodes Distribution for Coexisting Wireless Body Area Network Based on Stochastic Geometry
}

\author{
Ruixia Liu ${ }^{1 *}$, Yinglong Wang ${ }^{1}$, Minglei Shu ${ }^{1}$, Huiqi Zhao ${ }^{2}$ and Changfang Chen ${ }^{1}$ \\ ${ }^{1}$ Qilu University of Technology (Shandong Academy of Sciences), Shandong Computer Science Center (National \\ Supercomputer Center in Jinan), Shandong Provincial Key Laboratory of Computer Networks \\ Jinan 250014 - China \\ ${ }^{2}$ Department of Information Engineering, Shandong University of Science and Technology \\ Taian 271021 - China \\ [e-mail: liurx@sdas.org] \\ *Corresponding author: Ruixia Liu
}

Received September 21, 2017; revised January 3, 2018; accepted June 25, 2018;

published November 30, 2018

\begin{abstract}
The coexisting wireless body area networks (WBAN) is a very challenging issue because of strong inter-networks interference, which seriously affects energy consumption and spectrum utilization ratio. In this paper, we study a power control strategy with nearest neighbor nodes distribution for coexisting WBAN based on stochastic geometry. Using homogeneous Poisson point processes (PPP) model, the relationship between the transmission power and the networks distribution is analytically derived to reduce interference to other devices. The goal of this paper is to increase the transmission success probability and throughput through power control strategy. In addition, we evaluate the area spectral efficiency simultaneously active WBAN in the same channel. Finally, extensive simulations are conducted to evaluate the power control algorithm.
\end{abstract}

Keywords: Stochastic Geometry, Power Control, WBAN, Network Coexistence, Success Probability, Inter-networks Interference

This work is supported by the National Natural Science Foundation of China under Grant No. 61401256 and No. 61603224, the natural science foundation of Shandong Province under Grant No. ZR2017MF029 and by the Innovation Program of the Shandong Academy of Sciences Grant No. [2012]153. 


\section{Introduction}

Wireless body area networks (WBAN) has become an important technology to collect continuously health-related data for disease diagnosis. In WBAN, physiological signals, motion or environmental data are gathered by the wireless sensors placed on or implanted into the body and transmitted to a coordinator [1]. In many applications, multi-WBANs often coexist in a small area such as nursing home or playground, which lead to severe interference between networks. The interference caused by coexisting WBAN can significantly result in loss of data, energy consumption or time delay. However, network reliability is especially important in medical applications.

In general, sensor nodes in WBAN are typically battery-powered, and the battery lifetime is required to be up to several years [2]. Owing to the limited battery capacity in sensor nodes, effective power control is especially crucial in the design and communication reliability of WBAN. The transmission power of sensor nodes should be adjusted in an on-demand way to adapt to the changing surroundings, in order to enhance energy efficiency and reduce conflict to other devices.

One of the main sources of energy consumption in coexisting WBANs origins from inter-networks interference between nearby WBAN, resulting to a bad influence on network performance. Although obviously, the signal strength and the aggregate interference at a receiver critically depend on the spatial positions of the WBANs, especially the nearby nodes [3]. The mathematical techniques are needed to explicitly model the nodes distribution [4].

On one hand, the heterogeneous Poisson point process (PPP) model is the simplest and most important random point pattern of stochastic geometry for the location of nodes in networks when their position is unknown. It has been widely used to the analysis and design of wireless networks in particular. Stochastic geometry is an established branch of mathematics which studies uncertainty in geometric structures. Its theory has recently emerged as an essential tool to model and quantify interference which is verified to be close to the actual networks [5]. Recently, stochastic geometry has emerged as a powerful tool to analyze random networks adopting media access control (MAC) protocols such as ALOHA and carrier sense multiple access (CSMA). IEEE 802.15.4 protocol is considered as a promising technology for WBAN. On the basis of above considerations, we study the performance and the power control strategy of coexisting IEEE 802.15.4-based WBAN in this study. The major contributions of the work can be summarized as follows:

- A unified framework is developed for the design of coexisting model for IEEE 802.15.4-based WBAN. We analyze the interference of inter-networks using stochastic geometry. In addition, the success probability expression of the coexisting WBANs is derived.

- In order to reduce the completion or collision in coexisting WBANs, this paper proposes a new power control strategy based on the nearest neighbor nodes distribution. The interference is affected seriously by the nearby nodes. Therefore, we derive the relationship of transmission power based on the nearest neighbor nodes. We characterize two types of power control strategies: the single transmitter node power control and all the nodes transmission power control. The simulation results show that the inter-WBANs interference is decreased and the throughput is increased through this power control strategy.

- Furthermore, we analyze the area spectral efficiency of coexisting WBANs to 
measure the utilization of the allocated frequency bands. The number of transmitter nodes is estimated that can coexist in the same frequency channel. Then the appropriate density can be obtained in the same contain domain according to the area spectral efficiency.

In this paper, we study the effects of coexisting WBANs and propose the nearest neighbor nodes power control strategy based on stochastic geometry. We analyze two situations of power control strategies: single node optimal power control and all transmitter nodes power control. The remainder of this paper is organized as follows. Section 2 reviews the related work. The system model is introduced in Section 3. Section 4 formulates the power control and the number of coexisting WBANs in PPP model. While Section 5 describes and analyzes numerical results that show the effect of power control. Finally, concluding remarks are discussed in Section 6.

\section{Related Work}

In the following we describe the related works on power control of wireless networks. Two main schemes, which are optimization and game theory approaches, have been used to analyze and design efficient power control policies in wireless networks [6]. Advances in wireless sensor networks such as power supply miniaturisation, increased battery duration, reduced energy consumption, and power scavenging are essential to systems that undertake pervasive monitoring, particularly in regard to implantable sensors [7]. In [8], the authors developed a class of practical online schemes that dynamically adapt transmission power based on receiver feedback. Some other papers have investigated power optimization about MAC protocols such as in studies [9-12]. In [13], a new distributed queuing body area network MAC protocol commitment is proposed to guarantee that all packet transmissions are served with their particular application-dependant quality of service requirements.

Coexisting of multiple WBANs is a rather challenging problem because there is co-channel interference because of the high mobility or a certain density. The closest distance of inter-WBANs can be $10 \mathrm{~cm}$ according to paper [14]. Some other papers about interference-mitigated algorithms have been proposed for WBAN. In [15], an interference cancellation algorithm was proposed to mitigate the interference among devices in one WBAN with ultra wideband and multiple-input-multiple-output (MIMO) systems. Other works [16-17] have considered the coexistence with other wireless technologies.

Some papers have already noticed the impact of nearest neighbor nodes, but most of these topics are confined to the network connectivity or topology [18-19]. In [18], the authors analyzed the connectivity of the wireless sensor networks based on the nearest neighbor nodes distance. A novel approach in [19] was proposed to detect spatial cluster of points according to the nearest neighbor distance. A nearest-neighbor clustering power control algorithm was proposed in [20]. The transmission power of nodes in the same cluster were optimized by according to the nearest-neighbor distances algorithm. It can reduce network latency time, prolong network lifetime. But this algorithm must know the number of cluster beforehand.

In [21], a distributed algorithm was proposed which minimized the power required for neighbor discovery. In a high node density networks, the lifetime of the network increased by using the optimal scheme during each instance of neighbor location. The process of neighbor nodes discovery is modeled as a Markov decision process. However, there were many remaining open questions such network discovery when nodes failed or moved.

A new, adaptive and energy-efficient MAC protocol based on multi-WBANs was proposed in [22]. This model can prevent collisions and prolong the network lifetime by 
rotating the coordinator node and WBAN member nodes, by using transmission power adjustment approach and using channel hopping. However, the WBAN nodes were set to the lowest possible transmission power.

The author studied that a connected network could be generated through assigning communication radius to each of them in [23]. These papers, generally speaking, try to guarantee a connected network or a certain topology to achieve better performance through adjusting transmission power. However, the connectivity was studied on the basis of distance between receiver and transmitter, not considering about mutual interference.

Unlike most of the other works listed above, we allow the transmission power at each node to be a random variable with arbitrary distribution subject to the density of coexisting WBANs under the constrained of power. The results of this paper show that this strategy is especially suited for the coexisting of multiple homogeneous wireless networks.

\section{System Model}

\subsection{Poisson Point Process Model}

A coordinator node and several sensor nodes form a WBAN. The coordinator node, such as mobile phone, is placed at center of the human body. Some sensor nodes are deployed on different parts of body according to different monitoring parameters. All these wireless nodes build a star network topology. In this star topology, the communication is established between sensor nodes and the coordinator. Multi-WBANs are modeled as a stationary Poisson cluster process coexisting in the $\mathbb{R}^{2}$ Euclidean space illustrated in Fig. 1.

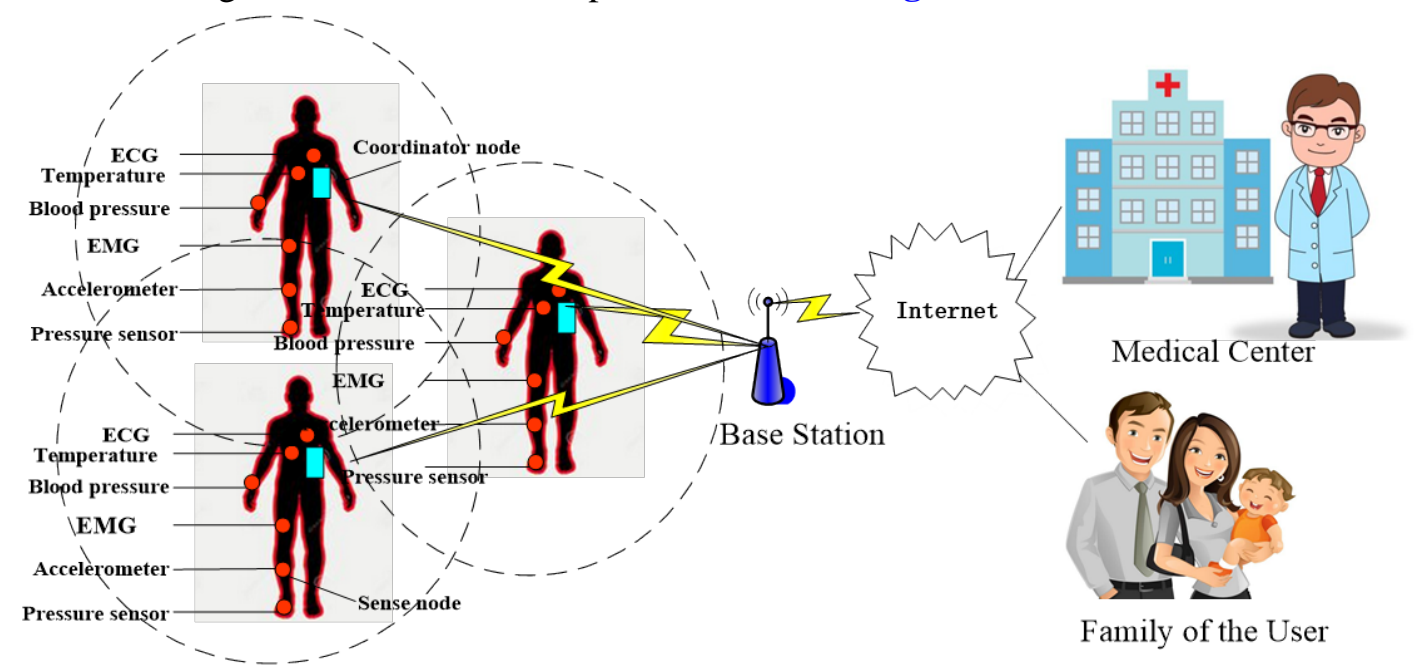

Fig. 1. Coexisting WBANs model

In this paper, we consider that each WBAN has a node sending data at the same time according to the competition of CSMA scheme. That is, every transmitter $\Psi=\left\{\left(\chi_{i}, d_{i}\right) ; i=1,2,3, \ldots\right\}$ is independently and randomly distributed in $\mathbb{R}^{2}$ Euclidean space according to an homogenous Poisson point process with intensity $\lambda$, where $\chi_{i} \in \Psi$ denotes the location of the $\boldsymbol{i}^{\text {th }}$ transmitter node and $\boldsymbol{d}_{\boldsymbol{i}}$ denotes the distance of the nearest neighbor nodes. We adopt the following assumptions and definitions: 
- It is assumed that all the sensor nodes have the same maximum transmission power of $P_{\max }$ and the minimum $P_{\min }$ at first.

- The interference domain $R_{I}$ for each receiver node is the range of the maximum distance from which a receiving node can sense a carrier. It depends on the transmission power of the sender and the other near neighbor nodes.

- The channel of all the coexisting WBANs accessing is the same logical channel.

\subsection{Radio Channel Model}

In this paper, the path-loss attenuation effect is only considered and additional channel influence is ignored such as shadowing and fast fading. In particular, a general path-loss model is considered as $r^{-\eta}$ with the propagation distance $r$. The received power at a distance $r$ from the transmitter is $P_{t} h r^{-\eta}$ on a single link if there is no outside interference [24], where the $P_{t}$ is the transmission power. The $\eta$ is the path-loss exponent and $h$ is the power fading coefficient which follows an exponential distribution with mean $1 / \mu$, written as $h \sim \exp (\mu)$.

\section{Power Control Strategy}

\subsection{Modeling the Aggregate Interference and Success Probability}

We first give a general expression for the interference in coexisting WBANs whose transmitter nodes are distributed as a stationary PPP model with density $\lambda$. Without loss of generality, we may take a coordinator node to be at the origin $O$ according to the Slivnyak theorem of the stochastic geometry [5]. The sensor nodes $x_{i}$ is located in the coordinator node's interference domain $\left\|x_{i}\right\|<R_{I}$. The total interference seen by a receiver is the sum of the signal powers received from all transmitters, except its own transmitter. Therefore, the cumulative interference at the origin, which is caused by all other transmitter nodes $x_{i}$ located in the contain domain $\phi_{\mathcal{L}}$, can be defined as

$$
I=\sum_{x \in \phi_{\mathcal{L}}} P_{i} h_{i}\left\|x_{i}\right\|^{-\eta}
$$

where the $P_{i}$ is the transmission power of node, $h_{i}$ is the fading coefficient of $x_{i}$.

We consider the following signal to interference and noise (SINR) model for a receiver placed at the origin $O$ in the two Euclidean space is [25]

$$
S I N R_{s}=\frac{P_{t} h r_{0}^{-\eta}}{N_{0}+I}
$$

where $r_{0}$ is the distance from the sending node to the origin coordinator node transmission distance, $P_{t}$ is the transmitter power of this sensor node, $h$ is the fading coefficient. $I$ is the cumulative interference of the origin node's and $N_{0}$ is noise power. 
The packet can be successful transmitted if the SINR exceeds a certain threshold $\beta$. So we define the success probability as follows

$$
\rho_{s}=\mathbb{P}\left(\frac{P_{t} h r_{0}^{-\eta}}{N_{0}+I} \geq \beta\right)
$$

\subsection{Power Control Strategy}

Power management is always a very important issue in Ad hoc networks, wireless sensor networks, as well as WBAN [26]. In the coexisting WBANs, the distribution of nodes is random and cannot be predicted owing to the mobility of people. It requires a mechanism to change the transmission power according to the distribution of nodes. It can not only reduce the total energy consumption of the network, but also overcome the main limiting factor of interference. The transmission capacity of the whole networks, moreover, can be improved [27].

The geographical locations of nodes are a major influencing factor of the performance of WBAN coexistence. The PPP distribution satisfies the randomness of the networks. Furthermore, it is the simplest model and plays a fundamental role in random point pattern. The interference created by other sending nodes will change with the variety of network density, especially affected by neighbor nodes. Because of these, we focus only on the first nearest neighbor nodes and ignore the second nearest neighbor nodes distribution in this paper due to the characteristics of short range communication of WBAN.

The cumulative complementary distribution function (CCDF) of the nearest point of the process from the origin denoted by $d$ is [5]

$$
\mathbb{P}(d \geq \mathrm{r})=\exp \left(-\lambda \pi \mathrm{r}^{2}\right)
$$

The nearest neighbor nodes distance distribution

$$
f(d)=2 \pi \lambda d \exp \left(-\pi \lambda d^{2}\right)
$$

The success probability $\rho_{s}$ of WBANs coexistence, which is the same as the cumulative distribution function (CDF) of the SINR, can be expressed as

$$
\begin{aligned}
\rho_{s} & =\mathbb{P}\left(\frac{P_{t} h r_{0}^{-\eta}}{N_{0}+I} \geq \beta\right) \\
& =\mathbb{P}\left\{\mathrm{h} \geq\left(\frac{\beta N_{0} r_{0}^{\eta}}{\mu P_{t}}+\frac{\beta r_{0}{ }^{\eta} I}{\mu P_{t}}\right)\right\} \\
& =\mathbb{E}_{r}\left\{\exp \left(-\frac{\beta N_{0} r_{0}{ }^{\eta}}{\mu P_{t}}\right) \mathbb{E}\left[\exp \left(-\frac{\beta r_{0}{ }^{\eta} I(r)}{\mu P_{t}}\right) \mid r\right]\right\} .
\end{aligned}
$$

Given $\mathcal{L}_{p}(s)=\mathbb{E}\left[\exp \left(-\frac{\beta r_{0}{ }^{\eta} I(r)}{P_{t}}\right)\right] \quad s=\frac{\beta r_{0}{ }^{\eta}}{P_{t}}$, $g(x)=\frac{1}{\sqrt{3}} \arctan \frac{2 x-1}{\sqrt{3}}-\frac{1}{6} \ln \frac{(1+x)^{2}}{1-x+x^{2}}-\frac{1}{\sqrt{3}} \arctan \left(-\frac{1}{\sqrt{3}}\right)$ The Laplace transform of 
interference

$$
\mathcal{L}_{p}(s)=\mathbb{E}\{\exp (-s I(r))\}=\exp \left\{-\lambda\left(\frac{\beta r_{0}{ }^{\eta}}{P_{t}} \hat{P}_{t}\right)^{2 / \alpha}[g(\mathrm{r})-g(0)]\right\}
$$

See Appendix A.

\subsection{Single Node Power Control (SNPC)}

We first consider the case that only one node $x_{1} \in \phi$ can use power control but all other transmitter nodes with certain value. Therefore the $S I N R_{s}$ at the receive node is

$$
\operatorname{SINR}_{\mathrm{s}}=\frac{\widehat{P}_{t} h r_{0}^{-\eta}}{N_{0}+I}
$$

where $\widehat{P}_{t}$ is the least required transmission power to achieve the target SINR $\beta$.

$$
\begin{aligned}
& \rho_{s}=\mathbb{P}\left(\frac{\widehat{P}_{t} h r_{0}^{-\eta}}{N_{0}+I} \geq \beta\right) \\
= & \mathbb{P}\left\{\mathrm{h} \geq\left(\frac{\beta N_{0} r_{0}^{\eta}}{\mu \widehat{P}_{t}}+\frac{\beta r_{0}{ }^{\eta} I}{\mu \widehat{P}_{t}}\right)\right\} \\
= & \mathbb{E}_{r}\left\{\exp \left(-\frac{\beta N_{0} r_{0}{ }^{\eta}}{\mu \widehat{P}_{t}}\right) \mathbb{E}\left[\exp \left(-\frac{\beta r_{0}{ }^{\eta} I(\mathrm{r})}{\mu \widehat{P}_{t}}\right) \mid \mathrm{r}\right]\right\} \\
= & \int_{0}^{d} f(\mathrm{r}) \exp \left(-\frac{\beta N_{0} r_{0}{ }^{\eta}}{\widehat{P}_{t}}\right) \exp \left(-\lambda \int_{\mathbb{R}^{2}} \frac{1}{1+\left(s P_{t}\right)^{-1} x^{\eta}} d x\right) d r \\
= & \exp \left(-\frac{\beta N_{0} r_{0}{ }^{\eta}}{\widehat{P}_{t}}\right) \exp \left(-\lambda\left(\frac{\beta r_{0}{ }^{\eta}}{\widehat{P}_{t}} P_{t}\right)^{2 / \eta} \int_{0}^{d} f(\mathrm{r})[g(\mathrm{r})-g(0)] d r\right)
\end{aligned}
$$

The success probability of transmission is an expression related to the distance $d$ of the neighbor nodes. So our goal is to maximize the success probability of achieving or exceeding the threshold $\delta$. We can express the transmission power under a certain limit of success probability, which can be expressed as

$$
\begin{aligned}
& \operatorname{minimize} \widehat{P}_{t} \\
& \text { subject to } \rho_{s}>\delta \\
& \qquad P_{\min }<\widehat{P}_{t}<P_{\max }
\end{aligned}
$$




\subsection{All Nodes Power Control (ANPC)}

Now, let us assume that each node $x_{i} \in \phi$ can dynamically control the transmission power it uses independently of other nodes. $S I N R_{A}$ at the reference receiver can be written as

$$
S I N R_{A}=\frac{\widehat{P_{t}} h r_{0}^{-\eta}}{N_{0}+I_{A}}
$$

Thus, for an power control policy characterized by the random variable $\widehat{P}_{t}$, the successful probability can be expressed as

$$
\begin{aligned}
& \rho_{s}=\int_{0}^{d} f(\mathrm{r}) \exp \left(-\frac{\beta N_{0} r_{0}^{\eta}}{\widehat{P}_{t}}\right) \exp \left(-\lambda \int_{\mathbb{R}^{2}} \frac{1}{1+\left(s \mathbb{E}\left(P_{t}\right)\right)^{-1} x^{\eta}} d x\right) d r \\
& =\exp \left(-\frac{\beta N_{0} r_{0}^{\eta}}{\widehat{P}_{t}}\right) \exp \left\{-\lambda\left(\frac{\beta r_{0}^{\eta}}{\widehat{P}_{t}} \mathbb{E}\left(P_{t}\right)\right)^{2 / \eta}\right\} \int_{0}^{d} f(\mathrm{r}) \exp \{-[g(\mathrm{r})-g(0)]\} d r \\
& =c \exp (-\mathrm{a}) \exp \left(-b \mathbb{E}\left(P_{t}\right)\right)^{2 / \eta}
\end{aligned}
$$

Where $a=\frac{\beta N_{0} r_{0}{ }^{\eta}}{\widehat{P}_{t}}, b=\lambda\left(\frac{\beta r_{0}{ }^{\eta}}{\widehat{P}_{t}}\right)^{2 / \eta}, c=\int_{0}^{d} f(\mathrm{r}) \exp \{-[g(\mathrm{r})-g(0)]\} d r$.

According to the success probability threshold $\delta$, we seek the mean transmission power value $\mathbb{E}\left(P_{t}\right)$ allocation on each sensor node that achieves the minimum $I_{A}$, the success probability (11) becomes

$$
\left.\rho_{s}=c \exp (-a) \exp \left(-b \mathbb{E}\left(P_{t}\right)\right)^{2 / \eta}\right)>\delta
$$

From (12), we can obtain

$$
\begin{aligned}
\exp \left(-b\left(\mathbb{E}\left(P_{t}\right)\right)^{2 / \eta}\right) & >\frac{\delta}{c} \exp (a) \\
-b\left(\left(\mathbb{E}\left(P_{t}\right)\right)^{2 / \eta}\right) & >a \ln \left(\frac{\delta}{c}\right) \\
\left(\mathbb{E}\left(P_{t}\right)\right)^{2 / \eta} & <-\frac{a}{b} \ln \left(\frac{\delta}{c}\right) \\
\mathbb{E}\left(P_{t}\right) & <\left(-\frac{a}{b} \ln \left(\frac{\delta}{c}\right)\right)^{2 / \eta}
\end{aligned}
$$

Then, the transmission power with constraints is

$$
\widehat{P}_{t}= \begin{cases}\min \left\{\left(-\frac{a}{b} \ln \left(\frac{\delta}{c}\right)\right)^{2 / \eta}, P_{\max }\right\} & \left(-\frac{a}{b} \ln \left(\frac{\delta}{c}\right)\right)^{2 / \eta}>P_{\min } \\ P_{\min } & \left(-\frac{a}{b} \ln \left(\frac{\delta}{c}\right)\right)^{2 / \eta} \leq P_{\min }\end{cases}
$$




\subsection{The Area Spectral Efficiency of Coexisting WBANs}

The wireless sensor networks related technologies primarily operate in the unlicensed industrial scientific medical (ISM) band which is shared with other major wireless standards such as IEEE 802.11, Bluetooth, and cordless phone. With the growing proliferation of wireless devices and systems, the ISMband is increasingly becoming congested and the coexistence issues are becoming more and more critical for the applications of wireless sensor networks. Many standard solutions for WBAN operate in this ISN band centered at $2.45 \mathrm{GHz}$ [28]. Therefore, we should consider about the spectrum efficiency.

In this section, we discuss the area spectral efficiency which is defined as the utilization of channel. We evaluate the area spectral efficiency based on the maximum number of sending nodes at a time in the same channel. Note that the area spectral efficiency can be optimized through increasing the density of WBAN which can operate in a logical channel. According to the formula (3), we can obtain that the number of area simultaneously active WBAN access the same logical channel is $\lambda \rho_{s}$. The different number of active WBAN can be obtained in the light of different success probability under the power control. According to the maximum value $\lambda \rho_{s}$, we can get the most suitable density of WBANs coexistence.

\section{Simulation Results}

This section evaluates the performance of the proposed power control schemes through Monte Carlo simulations in MATLAB. We have distributed many wireless nodes in the $20 \mathrm{~m}$ times $20 \mathrm{~m}$ area according to a certain density $\lambda$ as a two-dimensional homogeneous PPP model. The interference region of every receiver node is changed in the light of variety of transmission power $P_{t}$. If the nearest neighbor nodes distance is less than the interference range of a receive node, there will cause very strong inter-WBANs interference. To simplify the analysis, we exploit the fact that, each sending node can only transmit data to its coordinator of the same WBAN according to the IEEE 802.15.4 standard. The distance between them is $r_{0}$. Table 1 summarizes the parameters and their values according to CC2530 radio and IEEE 802.1.5.4 technology used in our simulations [29].

Table 1. System parameters

\begin{tabular}{|c|c|}
\hline On-body path-loss exponent $\eta$ & 3 \\
\hline SINR threshold $\beta(\mathrm{dB})$ & -15 \\
\hline The power of the minimum $P_{\min }(\mathrm{dBm})$ & -25 \\
\hline The power of the maximum $P_{\max }(\mathrm{dBm})$ & 0 \\
\hline Channel bandwidth $(\mathrm{Mhz})$ & 2 \\
\hline Frequency $(\mathrm{Ghz})$ & 2.4 \\
\hline Channel rate $(\mathrm{kbps})$ & 200 \\
\hline
\end{tabular}




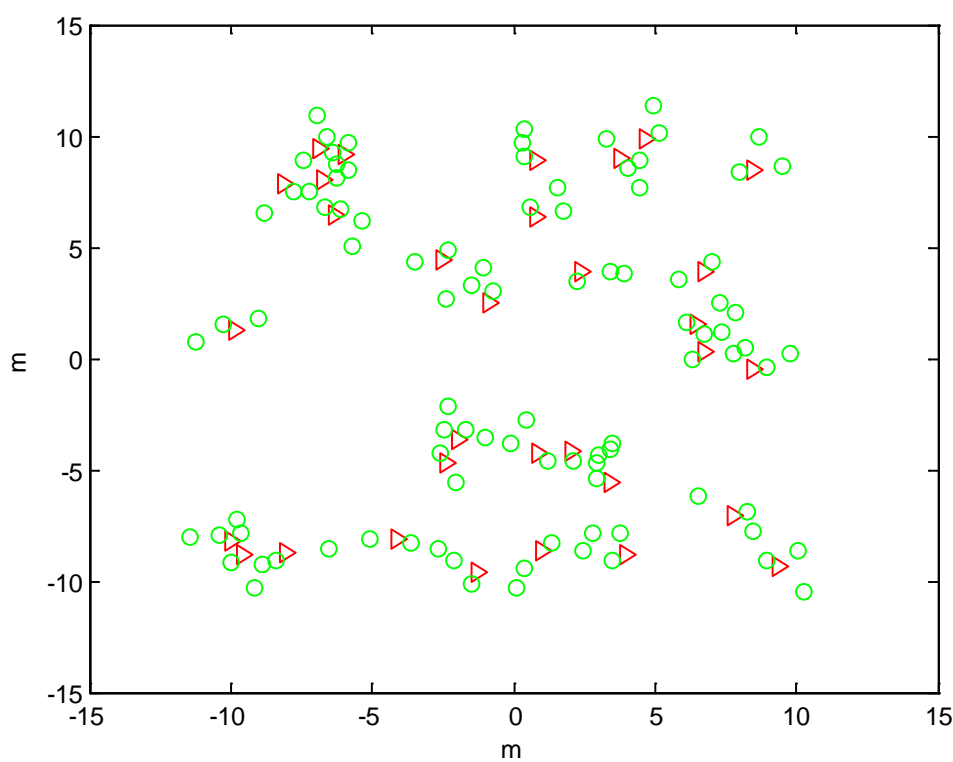

(a)

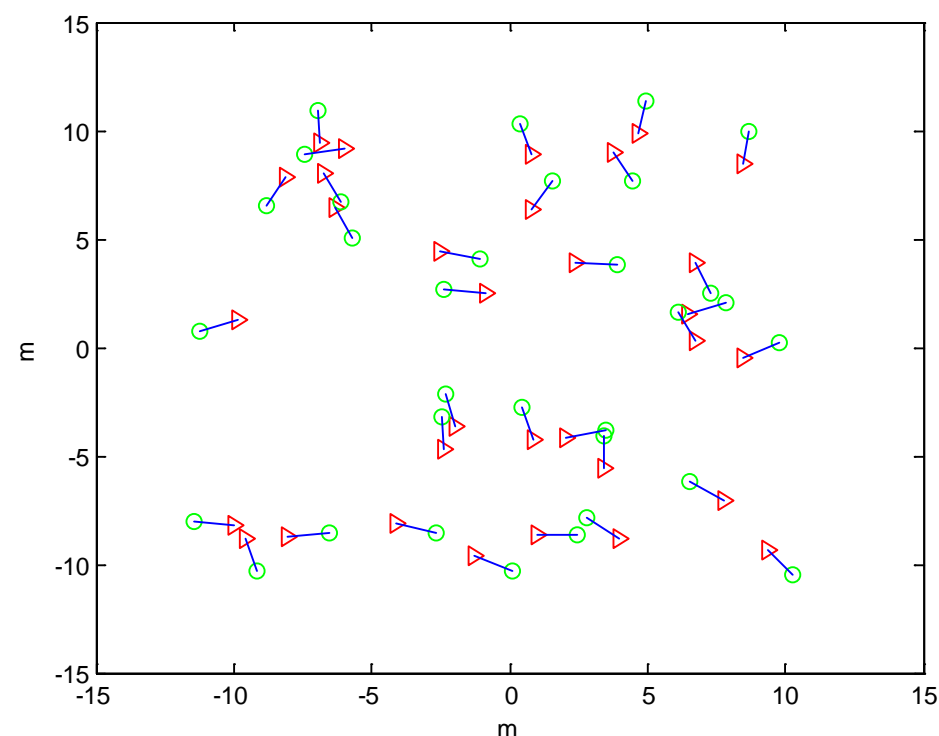

(b)

Fig. 2. PPP model (Red triangle symbol represents the Coordinator node while the green round dots represents the sensor nodes.)

The coexisting networks model considered in this paper are composed of multiple stationary WBANs which have a receive node (Coordinator node) and several sensor nodes randomly located within a certain distance in the $\mathbb{R}^{2}$ Euclidean space as shown in Fig. 2 (a). To simplify the analysis, we analyze one sensor node and one receive node in each WBAN according to the IEEE 802.15.4-based CSMA in the beacon enabled mode such as Fig. 2 (b). Therefore, the density $\lambda$ of sensor nodes is also the density of WBANs. 


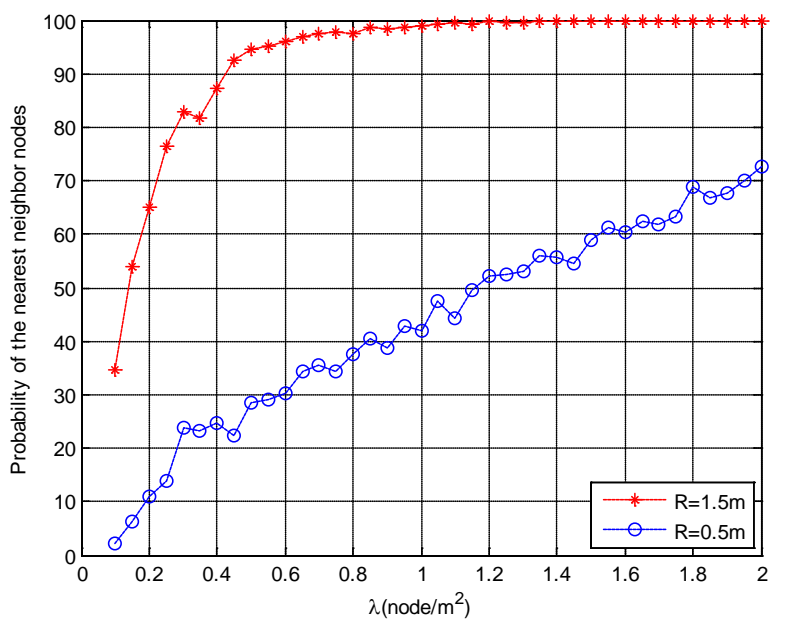

Fig. 3. The probability of the node has the first nearest neighbor nodes

Fig. 3 illustrates the simulation results of the probability distribution that a node has the first nearest neighbor under the different interference region. The difference of the probability is remarkable although the interference region changes few. It would contribute to the analysis of power control's significance if each node is capable of finding nearest neighbor nodes and adjusting transmitting range according to local requirement.

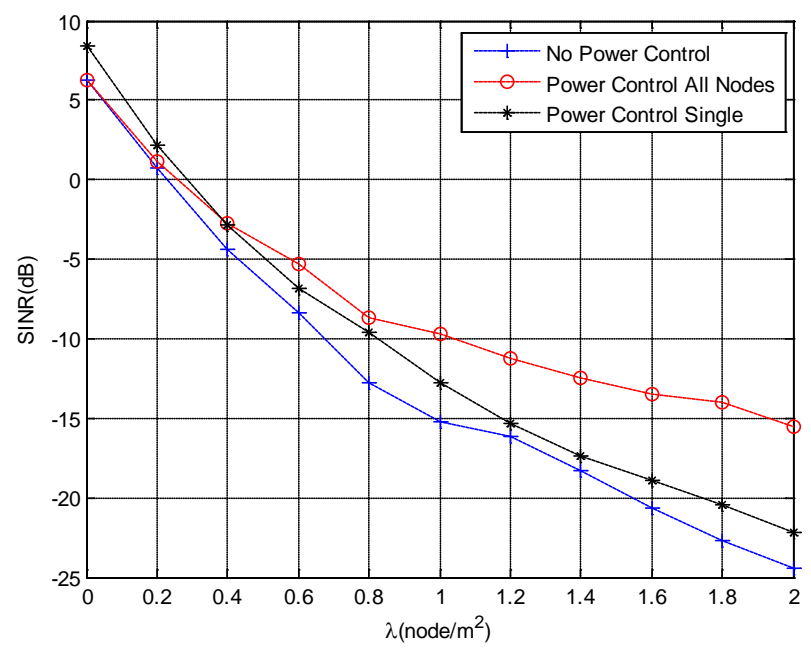

Fig. 4. SINR

The SINR on a wireless link is an important basis for consideration of outage, capacity, and throughput in a wireless network. It is therefore important to understand the SINR distribution within such networks [30]. From Fig. 4, we investigate the effect of power control to the link of SINR. However, increasing the density $\lambda$ to a certain level can cause the greater the variety of power control. Note that as it can be obviously seen for $\lambda>1$, the SINR of all nodes power control can be better than $-16 \mathrm{~dB}$. This shows that this power control strategy can improve the SINR of the coexisting WBANs. 


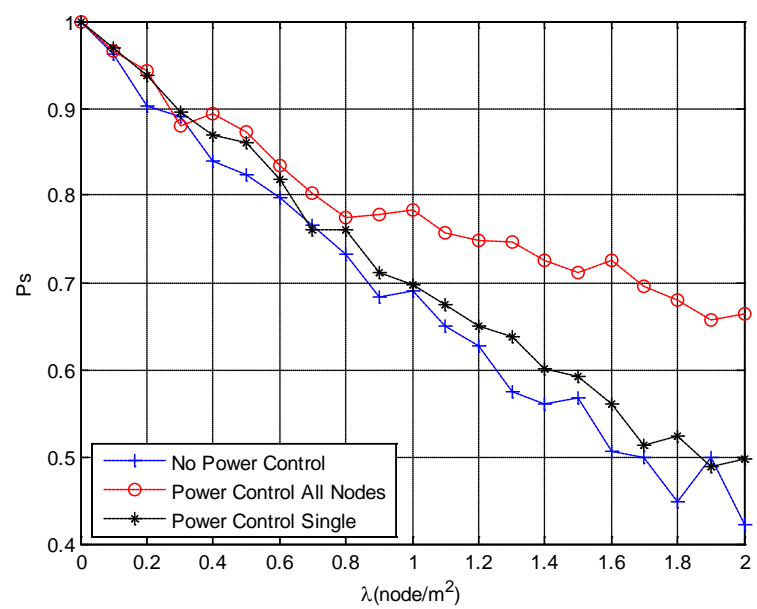

Fig. 5. The success probability

The success probabilities of three conditions are compared in Fig. 5. From the simulation results, the power control can carefully take advantage of the increase in the density. The success probability decline rapidly as the density increase, however, the power control can relieve slightly.

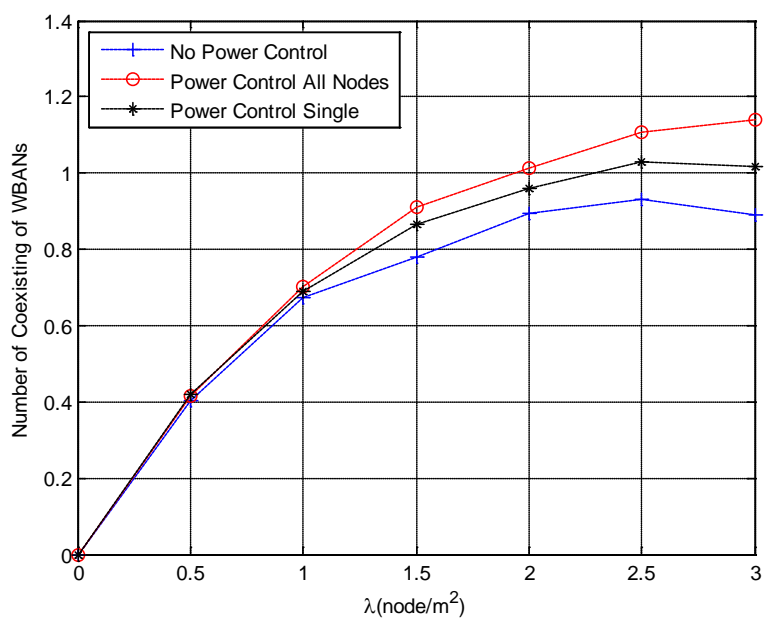

Fig. 6. The number of coexisting WBANs

Fig. 6 exhibits the number of coexisting WBANs under three conditions with the different density. It shows that there is a nonlinear relationship between number of channels and the density of WBANs coexistence. The power control about the neighbor nodes distance is excellent to the constant power and one single node power control. On the other hand, the number of the WBANs tends to be stable when the networks density is somewhat higher, and then it begins to decrease. This is due to the fact that, the outage probability $1-\rho_{s}$ is relatively big owing to the aggregate and conflict. According to the maximum value of the curve, we can get the most suitable density of WBANs coexistence. 


\section{Conclusion}

In this paper, we investigate the transmission power optimization for coexisting WBANs. Using stochastic geometry tools, we have derived an expression for the power control in two cases: 1) the single transmitter node power control and 2) all the nodes control. The inter-WBANs interference is decreased and the transmission success probability or the throughput is increased through power control scheme. Finally, extensive simulations are conducted with two scenarios to evaluate the power control.

\section{APPENDIX A:}

The interference component of the success probability can be calculated by determining the Laplace transform of $I$.

Given $\mathcal{L}_{p}(s)=\mathbb{E}[-s I(r)], s=\frac{\beta r_{0}{ }^{\eta}}{P_{t}}$ and using the change of variables $g(x)=\frac{1}{\sqrt{3}} \arctan \frac{2 x-1}{\sqrt{3}}-\frac{1}{6} \ln \frac{(1+x)^{2}}{1-x+x^{2}}-\frac{1}{\sqrt{3}} \arctan \left(-\frac{1}{\sqrt{3}}\right)$ the Laplace transform of interference, respectively become

$$
\begin{aligned}
\mathcal{L}_{p}(s) & =\mathbb{E}[\exp (-s I(r))] \\
& =\mathbb{E}\left[\exp \left(-s \sum_{x \in \phi_{\mathcal{L}}} P_{i} h_{i} r^{-\eta}\right)\right] \\
& \underline{\underline{(a)}} \mathbb{E} \prod_{x \in \phi_{\mathcal{L}}} \exp \left(-s P_{i} h_{i} r^{-\eta}\right) \\
& \underline{\underline{(b)}} \mathbb{E} \prod_{x \in \phi_{\mathcal{L}}} \frac{1}{1+s P_{i} r^{-\eta}} \\
& \underline{\underline{(c)}} \exp \left(-\lambda \int_{R^{2}}\left(1-\frac{1}{1+s \hat{P}_{t} r^{-\alpha}} d r\right)\right) \\
& =\exp \left(-\lambda \int_{R^{2}} \frac{1}{1+\left(s \hat{P}_{t}\right)^{-1} r^{\alpha}} d r\right)
\end{aligned}
$$




$$
\begin{aligned}
& =\exp \left(-\lambda\left(s \hat{P}_{t}\right)^{2 / \alpha} \int_{0}^{2 \pi} d \theta \int_{0}^{r} \frac{x}{1+x^{\alpha}} d x\right) \\
& =\left.\exp \left(-\lambda\left(s \hat{P}_{t}\right)^{2 / \alpha} g(x)\right)\right|_{0} ^{r} \\
& =\exp \left\{-\lambda\left(s \hat{P}_{t}\right)^{2 / \alpha} \frac{1}{\sqrt{3}} \arctan \frac{2 r-1}{\sqrt{3}}-\frac{1}{6} \ln \frac{(1+r)^{2}}{1-r+r^{2}}-\frac{1}{\sqrt{3}} \arctan \left(-\frac{1}{\sqrt{3}}\right)\right\} \\
& =\exp \left\{-\lambda\left(\frac{\beta r_{0}{ }^{\eta}}{P_{t}} \hat{P}_{t}\right)^{2 / \alpha} \frac{1}{\sqrt{3}} \arctan \frac{2 r-1}{\sqrt{3}}-\frac{1}{6} \ln \frac{(1+r)^{2}}{1-r+r^{2}}-\frac{1}{\sqrt{3}} \arctan \left(-\frac{1}{\sqrt{3}}\right)\right\} \\
& =\exp \left\{-\lambda\left(\frac{\beta r_{0}{ }^{\eta}}{P_{t}} \widehat{P}_{t}\right)^{2 / \alpha}[g(\mathrm{r})-g(0)]\right\} \\
& =\exp \left\{-\lambda\left(\frac{\beta r_{0}^{\eta}}{P_{t}} \hat{P}_{t}\right)^{2 / \alpha}\left[\frac{1}{\sqrt{3}} \arctan \frac{2 r-1}{\sqrt{3}}-\frac{1}{6} \ln \frac{(1+r)^{2}}{1-r+r^{2}}-\frac{1}{\sqrt{3}} \arctan \left(-\frac{1}{\sqrt{3}}\right)\right]\right\}
\end{aligned}
$$

(a) is obtained by the independence of the fading random variables.

(b) Follows from the Laplace transform of an exponential random variable.

(c) Follows by the probability generating functional of the PPP.

\section{References}

[1] A. Milenkovic, C. Otto and E. Jovanov, "Wireless sensor networks for personal health monitoring: Issues and an implementation,” Comput. Commun., vol. 29, no 13-14, pp. 2521-2533, 2006. Article (CrossRef Link)

[2] R. Liu, Y. Wang, S. Wu, CX. Wang and W. Zhang, "Energy efficiency and area spectral efficiency tradeoff for coexisting wireless body sensor networks,” Science China Information Sciences, vol. 59,no. 12, pp. 1-15, 2016. Article (CrossRef Link)

[3] R. Kazemi, R. Vesilo, E. Dutkiewicz and R. Liu, "Dynamic Power Control in Wireless Body Area Networks Using Reinforcement Learning With Approximation," in Proc. of IEEE 22nd International Symposium on Personal, Indoor and Mobile Radio Communication, pp. 2203-2208, 2011. Article (CrossRef Link)

[4] J. Andrews, F. Baccelli and R. Ganti, "A tractable approach to coverage and rate in cellular networks,” IEEE Trans. Commun, vol. 59, no. 11, pp. 3122-3134, 2010. Article (CrossRef Link)

[5] D. Stoyan, W. Kendall and J. Mecke , "Stochastic geometry and its applications,” 2nd. 1995.

[6] X. Zhang and M. Haenggi, "Random power control in Poisson networks," IEEE Trans. Commun., vol. 60, no. 9, pp. 2602-2611, 2012. Article (CrossRef Link)

[7] A. Shantaram, H. Beyenal, R. Raajan and A. Veluchamy, "Wireless sensors powered by microbial fuel cells,” Environmental Science and Technology, vol. 39, no. 13, pp. 5037-5042, 2005. Article (CrossRef Link)

[8] X. Shuo, A. Dhamdhere, V. Sivaraman and A. Burdett, "Transmission power control in body area sensor networks for healthcare monitoring,” IEEE J. Sel. Areas Commun., vol. 27, no. 1, pp. 37-48, 2009. Article (CrossRef Link)

[9] O. Omeni, A. Wong and A. Burdett, “Toumazou C. Energy efficient medium access protocol for wireless medical body area sensor networks,” IEEE Trans. Biomed. Circuits Syst., vol. 2, no. 4, pp. 251-259, 2008. Article (CrossRef Link)

[10] H. Li and J. Tan, "Heartbeat-driven medium-access control for body sensor networks," IEEE Trans. Inf. Technol. Biomed., vol. 14, no. 1, pp. 44-51, 2010. Article (CrossRef Link) 
[11] S. Rezvani and S. Ghorashi, "Context aware and channel-based resource allocation for wireless body area networks,” IET Wirel. Sens. Syst., vol. 3, no. 1, pp. 16-25, 2013. Article (CrossRef Link)

[12] F. Bouabdallah, N. Bouabdallah and R. Boutaba, "Efficient reporting node selection-based MAC protocol for wireless sensor networks,” Wirel. Netw., vol. 19, no. 3, pp. 373-391, 2013. Article (CrossRef Link)

[13] O. Begonya, A. Luis and V. Christos, "Highly reliable energy-saving MAC for wireless body sensor networks in healthcare systems," IEEE J. Sel. Areas Commun., vol. 27, no. 4, pp. 553-565, 2009. Article (CrossRef Link)

[14] L. Wang, C. Goursaud, N. Nikaein, L. Cottatellucci and J. Gorce, "Cooperative Scheduling for Coexisting Body Area Networks,” IEEE Trans. Commun., vol. 12, no.1, pp. 123 - 133, 2013. Article (CrossRef Link)

[15] J. Bae, Y. Choi and J. Kim, " Efficient interference cancellation scheme for wireless body area network,” Journal of Communications and Networks, vol. 13, no. 2, pp. 167-174. 2011. Article (CrossRef Link)

[16] X. Zhang and GS. Kang, "Cooperative carrier signaling: harmonizing coexisting WPAN and WLAN devices,” IEEE/ACM Trans. Netw., vol. 21, no. 2, pp. 426-439, 2013. Article (CrossRef Link)

[17] R. Francisco, L. Huang and G. Dolmans, "Coexistence of WBAN and WLAN in medical environments," in Proc. of the IEEE VTC 2009-Fall, pp. 1-5, 2009. Article (CrossRef Link)

[18] Y. Qu, J. Fang and S. Zhang, "Nearest neighbor nodes and connectivity of three-dimensional wireless sensor networks with Poisson point field," in Proc. of IEEE International Conference on Computer Science \& Information Technology, pp. 269-272, 2010. Article (CrossRef Link)

[19] A. Bar-Hen, M. Emily and N. Picard, "Spatial cluster detection using nearest neighbor distance," Spatial Statistics, vol. 14, pp. 400-411, 2015. Article (CrossRef Link)

[20] Y. Chen, Z. Wang, B. Liu and L. Ge, "Nearest-Neighbor Clustering Power Control Algorithm Wireless Sensor Networks,” Springer Berlin Heidelberg, vol. 216, pp. 545-551, 2011. Article (CrossRef Link)

[21] R. Madan, S. Lall, "An Energy-Optimal Algorithm for Neighbor Discovery in Wireless Sensor Networks," Mobile Networks and Applications, vol. 11, no. 3, pp. 317-326, 2006. Article (CrossRef Link)

[22] I. Kirbas, A.Karahan, A. Sevin and C. Bayilmis, "isMAC: An Adaptive and Energy-Efficient MAC Protocol Based on Multi-Channel Communication for Wireless Body Area Networks," Ksii Transactions on Internet \& Information Systems, vol. 7, no. 8, pp. 1805-1824, 2013. Article (CrossRef Link)

[23] M. Korman M, "Minimizing interference in ad hoc networks with bounded communication radius," Information Processing Letters, vol. 112, pp. 748-752, 2012. Article (CrossRef Link)

[24] M. Haenggi, "Local Delay in Poisson Networks with and without Interference," Communication, Control, \& Computing, pp. 1482-1487, 2010. Article (CrossRef Link)

[25] M. Haenggi and RK. Ganti, "Interference in large wireless networks," Now Publishers Inc., vol. 3, no. 2, pp. 127-248, 2009. Article (CrossRef Link)

[26] R. Cavallari, F. Martelli, R. Rosini, C. Buratti and R. Verdone, "A Survey on Wireless Body Area Networks: Technologies and Design Challenges," IEEE Communications Surveys and Tutorials, vol. 16, no. 3, pp. 1635-1657, 2014. Article (CrossRef Link)

[27] R. Liu, Y. Wang, M. Shu and S. Wu, "Throughput assurance of wireless body area networks coexistence based on stochastic geometry,” Plos One, vol. 12, no. 1, pp. 1-22, 2017. Article (CrossRef Link)

[28] S. Tang, Y. Zhang, L. Zhang and R. Yu, "Spectrum-Efficient wireless sensor networks," International Journal of Distributed Sensor Networks, 2015, pp. 1-2, 2015. Article (CrossRef Link)

[29] AH. Sodhro, Y. Li and MA. Shah, "Energy-efficient adaptive transmission power control for wireless body area network,” IET Communications, vol. 10, no. 1, pp. 81-90, 2016. Article (CrossRef Link) 
[30] S. Mukherjee, “Distribution of downlink SINR in heterogeneous cellular networks,” IEEE J. Sel. Areas Commun., vol. 30, no. 3, pp. 575-585, 2012. Article (CrossRef Link)

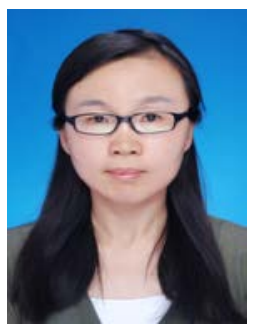

Ruixia Liu received the M.S. degree from the Shaanxi University of Science and Technology in 2004, respectively, the Ph.D. degree in information science and engineering from Shandong University of Science and Technology, Qingdao, China, in 2017. She has been a Associate Research with the Shandong Computer Science Center (National Supercomputer Center in Jinan). Her research interests include wireless body area networks for medical applications, the optimization of MAC protocol solutions, cross-layer optimization and Qos features.

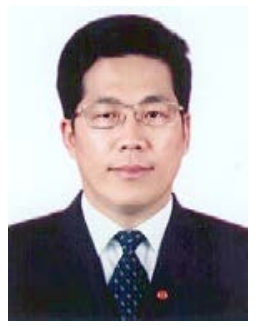

Yinglong Wang received the M.S. degree in industrial automation and the Ph.D. degree in communication and information systems from Shandong University, Jinan, China, in 1990 and 2005, respectively. He is a Research Fellow with the Shandong Computer Science Center (National Supercomputer Center in Jinan). His current research interests include wireless networks, information security, and cloud computing.

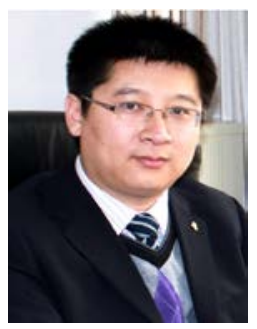

Minglei Shu received the M.S. degree in power electronics from Shandong University, Jinan, China, in 2006, and the Ph.D. degree in communication and information systems from Shandong University, Jinan, China, in 2016. He has been a Research Fellow with the Shandong Computer Science Center (National Supercomputer Center in Jinan). His research interests include wireless sensor networks, wireless body area networks, and information security.

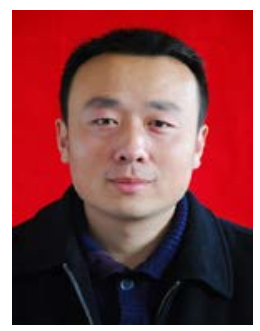

Huiqi Zhao received the B.S. and M.S. degree from Shandong University of Science and Technology, China in 2003 and 2009 respectively. He is currently working toward his Ph.D. degree in Shandong University of Science and Technology. His research interests are computer networks, wireless body area networks and healthcare applications, data privacy protection.

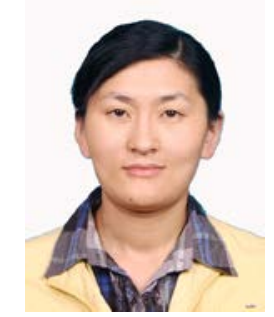

Changfang Chen received the B.S. degree in mathematics and the M.S. degree in control theory from Qufu Normal University, Qufu, China, in 2005 and 2008, respectively, and the $\mathrm{Ph}$.D. degree in control theory and applications from Beihang University (BUAA), Beijing, China, in 2013. From 2017 she was a Associate Research at Shandong Computer Science Center (National Supercomputer Center in Jinan). Her research interests include nonlinear and adaptive control with their applications in vehicle systems, wireless sensor networks, and wireless body area networks. 